\title{
Evaluation of clinical implications in the use of dose to water versus dose to medium by using NTCP and TCP models for urinary bladder tumours
}

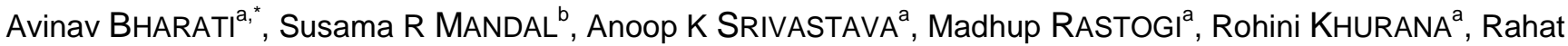 \\ $\mathrm{HADI}^{\mathrm{a}}$, Ajeet K GANDHI ${ }^{\mathrm{a}}$, Lalatendu MISHRA ${ }^{\mathrm{a}}$, Surendra P MISHRA ${ }^{\mathrm{a}}$ \\ ${ }^{a}$ Dr Ram Manohar Lohia Institute of Medical Sciences, Lucknow, India \\ ${ }^{b}$ Government Medical College, Kannauj, India \\ "E-mail address: avinavb5@gmail.com
}

\begin{abstract}
Purpose: To analyze the dosimetric and radiobiological differences between dose to water versus dose to medium for patients with carcinoma of the urinary bladder.

Materials and Methods: 15 patients with cancer of urinary bladder were selected for the study. VMAT plans were generated for each patient. The dose distributions were calculated in the modes dose to water and to medium with the Monaco treatment planning system. A dosimetric comparative analysis has been made between the two modes of planning in this study. Subsequently, NTCP and TCP were determined for OARs and targets respectively.

Results: The mean dose to $2 \mathrm{cc}$ of the rectum, small bowel, left and right femoral heads respectively was higher by 0.8 , $1.2,2.7$, and $2.2 \%$ for the dose to water calculation. Similarly, the mean dose to $\mathrm{D}_{2}, \mathrm{D}_{50}$, and $\mathrm{D}_{98}$ for PTV was higher by $0.4,0.3$, and $0.3 \%$ for dose to water calculation. Such small dose differences had little effect on the values of TCP and NTCP.

Conclusion: For patients with the urinary bladder there were very small differences between results between calculations carried out in dose to medium and dose to water modes.
\end{abstract}

Key words: NTCP; TCP; radiobiological; toxicity; dosimetry; dose to water; dose to medium.

\section{Introduction}

The accuracy of the dose calculation algorithm in heterogeneous medium has been a subject of interest among researchers for a very long time. Tissue inhomogeneity correction has undergone many changes and new methodologies have been upgraded and adopted to solve the problem. A dose calculation algorithm for high energy photon was first developed for a completely homogeneous medium and a patient, was considered to be composed entirely of water. In early $1969,{ }^{1}$ the dose computation in heterogeneous medium evolved purely empirically. Effective TAR (Tissue Air Ratio) method, linear attenuation coefficient method, and empirical methods of Batho $^{2}$ were employed to evaluate the doses in a heterogeneous medium. This class of algorithms was known as a correction-based algorithm. The advent of CT scanners allowed the implementation of the new class of dose distribution calculation methods. This brought spectral changes in the forms of dose computation algorithms. A model-based algorithm takes into consideration the real physical interaction of photon beams with the medium. The model-based algorithm requires greater calculation times and also provides better spatial resolution. ${ }^{3}$ Recent advances in coding and computer hardware techniques have substantially reduced calculation time for all the algorithms, also for the Monte Carlo method. Several vendors are currently supplying MC-based TPS. Currently, the Monte Carlo method is widely used in modern treatment planning systems. The energy deposition kernels used in convolution/ superposition have been calculated using $\mathrm{MC}$ techniques.

MC techniques have the capability of simulating and calculating the dose absorption directly to medium, accounting for the photon fluence and its interactions. They may not yield very different results for megavoltage photon beam (variations of $1-2 \%$ ) in soft tissue where the density of the medium is close to water i.e., $1 \mathrm{~g} / \mathrm{cc}$. But the difference may be significant up to $10 \%$ for high-density tissues like cortical bone. ${ }^{4}$ From the clinical point of view this will result in a large difference of dose for $\mathrm{H} \& \mathrm{~N}$ tumours sites where the tumor is embedded in the complex shape of a bone, soft tissue, and air cavities. In some treatment planning systems, the dose may be calculated to water or two medium. The two schools of thought have given arguments in favor and against these two methods of 
dose calculation. While some have simply advised following dose to water, to derive knowledge and compare with the past clinical practices. The method of calculation also influences the TCP and NTCP calculations for the tumor and the normal tissues. It should be also emphasized that measurements are based on ionization chamber calibration (for example AAPM's TG 51) ${ }^{5}$ protocols to dose to water. Moreover, tumor cells are always the water like.

On the other hand, the researchers in support of the method of calculations to medium $\left(\mathrm{D}_{\mathrm{m}}\right)$ have argued that the calculations to water $\left(D_{w}\right)$ is just an approximation for the actual conditions. The conversion of $D_{m}$ to $D_{w}$ is based on the Bragg-Gray cavity theory.

$D_{w}=D_{m}\left(\frac{\bar{S}}{\rho}\right) w, m$

Where $(\bar{S} / \rho) w, m$ is the unrestricted water to medium mass collision stopping power averaged over the energy spectra of primary electrons at the point of intent.

In this study, an attempt has been made to compare the dose difference between the calculation methods dose to water and dose to medium in case of cancer of the urinary bladder. The influence of these two methods on the TCP and NTCP have been also evaluated.

\section{Materials and Methods}

The study compares $\mathrm{D}_{\mathrm{m}}$ and $\mathrm{D}_{\mathrm{w}}$ in patients of Ca Urinary bladder calculated with Monaco treatment planning system. 15 randomly chosen patients of Ca urinary bladder were selected for this retrospective analysis. Patients underwent CT simulation using $3 \mathrm{~mm}$ thick slices with an empty bladder. The target and OAR delineation was performed as per institutional protocol and the dose constraints were provided by radiation oncologists. The radiation oncologist contoured rectum, bowel, femur and target volumes on the Monaco contouring station. The GTV and whole bladder were contoured to create CTV. A margin of $1 \mathrm{~cm}$ was given to the CTV to create PTV.

The selected cases were planned with statistical uncertainty per control point of $1 \%$, and the number of control points per field of 180, and the number of arcs per field of 2 . All the cases were planned with 2 full arcs, one clockwise and the other anticlockwise. The $6 \mathrm{MV}$ beams were used. All patients received $60 \mathrm{~Gy}$ in 30 fractions (5\# per week) to PTV primary using 6MV. The dose to the nodal PTV was $48 \mathrm{~Gy}$. The treatment plans were prepared with the Monaco system. First, a treatment plan was generated with $\mathrm{D}_{\mathrm{m}}$ based calculation, and subsequently, the plan was recalculated for the dose to water $\left(D_{w}\right)$ mode with exactly the same set of plan parameters (all the geometric parameters and monitor units (MU)) were retained.

After the plans had been generated the corresponding differential DVHs were saved and were transferred to the Biosuite. $^{6}$ The Biosuite is the radiobiological evaluation software. The NTCP for rectum, femoral heads, and intestine NTCP's were calculated using parameters (Table 1) available in the literature..$^{7-9}$ For the rectum the endpoints were rectal bleeding, proctitis, rectal urgency. Few other endpoints like small bowel toxicity and femoral head necrosis were evaluated for other OARs. For LQ Poisson model the TCPs ${ }^{10}$ were calculated with the LQ model.

\section{Results}

In Table 2 the average OAR dose received from plans calculated with the dose to water and medium is given. The dose to $0.1 \mathrm{cc}$ and $2 \mathrm{cc}$ volume of the organs at risk were measured for the two modes of calculation. Their average values were calculated for a total of 15 patients. The statistical analysis has been done manually using Student's t-test. Student's t-test is a commonly used technique for testing a hypothesis based on the difference between sample mean. The t-test determines the probability of whether two populations are the same with respect to the variable tested. This method is parametric and assumes the normality of the data and equality of variances across comparison groups. The relative percentage differences between the two values for each OAR and student $\mathrm{t}$-test value for $\mathrm{p}<0.05$ at 95 percent confidence is mentioned in Table 2.

Table 4 gives the student $t$ test calculation for $0.1 \mathrm{cc}$ volume of OARs Rectum, small bowel, Rt femur, Lt Femur in water and in medium. In order to test the hypothesis that the two samples belong to the same population the Student's t-test was used. The results are shown in Table $\mathbf{4}$ and can be seen that for all cases, $t$ is less than 2.048, which is the critical value of $t$ for $\mathrm{p}<0.05$. Hence the above hypothesis can be accepted with $95 \%$ confidence. The same result was obtained for $D_{2 c c}$ of OARs.

Table 1. Parameters used to calculate NTCP for Rectum, Bowel and femoral heads.

\begin{tabular}{|c|c|c|c|c|}
\hline Reference & Endpoint (Toxicity Grades) & TD50(1) (Gy) & $\mathbf{m}$ & $\mathbf{n}$ \\
\hline \multirow{6}{*}{ Gulliford et.al. $^{8}$} & Rectal bleeding (G1\&2) & 59.2 & 0.26 & 0.14 \\
\hline & Proctitis (G1\&2) & 58.2 & 10.28 & 0.14 \\
\hline & Rectal urgency (G1\&2) & 55 & 0.48 & 0.26 \\
\hline & Rectal bleeding (G2) & 68.2 & 0.14 & 0.12 \\
\hline & Proctitis $(\mathrm{G} 2)$ & 67.0 & 0.20 & 0.15 \\
\hline & Rectal urgency (G2) & 68. & 0.36 & 0.36 \\
\hline${\text { Burman et.al. }{ }^{9}}$ & Femoral Head (Necrosis) & 65 & 0.12 & 0.25 \\
\hline
\end{tabular}


Table 2. OAR Dose Analysis*

\begin{tabular}{|c|c|c|c|c|c|c|c|c|}
\hline OAR & $\begin{array}{c}\operatorname{Avg}_{0.1} D_{w} \\
0.1 c c\end{array}$ & $\begin{array}{c}\operatorname{Avg}_{2 c c} D_{w} \\
\end{array}$ & $\begin{array}{c}\operatorname{Avg}_{-} D_{m} \\
0.1 c c\end{array}$ & $\underset{2 c c}{\operatorname{Avg}_{-} D_{m}}$ & $\begin{array}{c}\text { Avg_\%Diff } \\
\text { (0.1cc)** }\end{array}$ & $\begin{array}{c}\text { Avg_\%Diff }_{(2 \mathrm{cc})} \\
\end{array}$ & $\begin{array}{c}t \text { value for } \\
0.1 \mathrm{cc}\end{array}$ & $\begin{array}{l}t \text { value for } \\
2 \mathrm{cc}\end{array}$ \\
\hline Rectum & $61.53 \pm 2.18$ & $58.55 \pm 3.13$ & $60.89 \pm 2.28$ & $57.73 \pm 2.87$ & $0.62 \pm 0.58$ & $0.80 \pm 1.44$ & 0.43 & 0.41 \\
\hline Small Bowel & $62.81 \pm 0.88$ & $60.86 \pm 1.66$ & $61.44 \pm 3.90$ & $59.65 \pm 4.49$ & $1.33 \pm 3.33$ & $1.22 \pm 3.81$ & 0.73 & 0.54 \\
\hline Lt Femur & $41.37 \pm 2.22$ & $36.34 \pm 2.65$ & $41.12 \pm 3.33$ & $36.25 \pm 2.67$ & $3.1 \pm 2.95$ & $2.7 \pm 2.6$ & 0.13 & 0.05 \\
\hline Rt Femur & $40.68 \pm 2.91$ & $35.38 \pm 3.02$ & $40.84 \pm 3.03$ & $35.36 \pm 3.08$ & $2.3 \pm 2.1$ & $2.2 \pm 2.1$ & 0.08 & 0.009 \\
\hline
\end{tabular}

*All the dose reported in Table 1 are in $G y$

**Avg_\%Diff $(0.1 c c)$ represents the average of the percentage difference of dose received by 0.1 cc of the OAR between the 2 modes of calculation i.e., $D_{w}$ and $D_{m}$. Similarly for Avg_\%Diff (2cc)

Table 3. PTV_primary Dose Analysis*

\begin{tabular}{|c|c|c|c|c|c|c|c|c|}
\hline $\begin{array}{c}\text { Avg_D } \\
\text { (Wat) }\end{array}$ & $\begin{array}{c}\text { Avg_D } \\
\text { (Wat) }\end{array}$ & $\begin{array}{c}\text { Avg_D } \\
\text { (Wat) }\end{array}$ & $\begin{array}{c}\text { Avg_D } \\
\text { (Med) }\end{array}$ & $\begin{array}{c}\text { Avg_D } \\
\text { (Med) }\end{array}$ & $\begin{array}{c}\text { Avg_D } \\
\text { (Med) }\end{array}$ & $\begin{array}{c}\text { Avg_\% Diff } \\
\left(\mathrm{D}_{2}\right)^{* *}\end{array}$ & $\begin{array}{c}\text { Avg_\% Diff } \\
\left(D_{50}\right)\end{array}$ & $\begin{array}{c}\text { Avg_\% }_{\left(D_{98}\right)} \\
\end{array}$ \\
\hline $63.43 \pm 0.24$ & $61.65 \pm 0.29$ & $58.66 \pm 0.49$ & $63.04 \pm 0.55$ & $61.39 \pm 0.37$ & $58.37 \pm 0.58$ & $0.37 \pm 0.43$ & $0.27 \pm 0.26$ & $0.30 \pm 0.32$ \\
\hline
\end{tabular}

*All the dose reported in Table 2 are in Gy

**Avg_\%Diff( $\left.D_{2}\right)$ represents the average of the percentage difference of dose received by $2 \%$ of the target volume between the 2 modes of calculation i.e., $D_{w}$ and $D_{m}$ Similarly for $A v g_{-} \% \operatorname{Diff}\left(D_{50}\right.$ and $\left.D_{98}\right)$

Table 4. Student's $t$ test calculation for Dose in water and Dose in Medium of OARs at $D_{0.1 c c}$ *

\begin{tabular}{|c|c|c|c|c|c|c|c|c|}
\hline \multirow[b]{2}{*}{ OAR } & \multicolumn{3}{|c|}{ Dose in water } & \multicolumn{3}{|c|}{ Dose in Medium } & \multirow[b]{2}{*}{$\begin{array}{c}\begin{array}{c}\text { Degree of } \\
\text { freedom }\end{array} \\
(r=n 1+n 2-2)\end{array}$} & \multirow[b]{2}{*}{ t value } \\
\hline & $\begin{array}{l}\text { Sample mean } \\
(\mathrm{m} 1)\end{array}$ & $\begin{array}{c}\text { Standard deviation } \\
\text { (s1) }\end{array}$ & $\begin{array}{l}\text { Sample size } \\
\text { (n1) }\end{array}$ & $\begin{array}{l}\text { Sample mean } \\
(\mathrm{m} 2)\end{array}$ & $\begin{array}{l}\text { Standard deviation } \\
(\mathrm{s} 2)\end{array}$ & $\begin{array}{c}\text { Sample size } \\
\text { (n2) }\end{array}$ & & \\
\hline Rectum & 61.53 & 2.18 & 15 & 60.89 & 2.28 & 15 & 28 & 0.43 \\
\hline Small Bowel & 62.81 & 0.88 & 15 & 61.44 & 3.90 & 15 & 28 & 0.73 \\
\hline Lt Femur & 41.37 & 2.22 & 15 & 41.12 & 3.33 & 15 & 28 & 0.13 \\
\hline Rt Femur & 40.68 & 2.19 & 15 & 40.84 & 3.03 & 15 & 28 & 0.08 \\
\hline
\end{tabular}

*All the dose reported in Table 3 are in Gy

For $r=28$, and significance $p>0.05$, the critical $t$ is $t_{0.975}=2.048$

If the absolute value of the $t$ value is greater than the critical value of $t_{0.975}$, then we reject the null hypothesis.

Table 3 gives the dosimetric analysis of the dose received by the PTV_primary. The dose received by 2, 50, and 98 percent $\left(\mathrm{D}_{2}, \mathrm{D}_{50}\right.$, and $\left.\mathrm{D}_{98}\right)$ of PTV_Primary were measured and averaged over 15 patients. The percentage difference between the measured values for the two modes was determined as given in Table 3. Student t values are 1.37, 1.17, and 0.81 for $\mathrm{D}_{2}, \mathrm{D}_{50}$, and $\mathrm{D}_{98}$ of PTV respectively. The above-obtained $\mathrm{t}$ values indicate that the null hypothesis that the sample belongs to the same group can be accepted with $95 \%$ confidence.

Table 5 provides the average NTCP obtained for various rectal toxicity endpoints for 15 patients for the two modes of calculation. Table 5 also gives the values of average NTCP for Small bowel toxicity and average TCP for 15 patients using the two modes of calculation. Student t-test value for $\mathrm{p}<0.05$ i.e., at 95 percent confidence interval is $0.242,0.169$ and 0.259 (grade 1 and 2 combined NTCP) for rectal bleeding, proctitis and rectal urgency respectively and the corresponding values for grade 2 toxicity were $0.164,0.155$ and 0.266 . Student t-test value for small bowel and PTV for $\mathrm{p}<0.05$ i.e., at 95 percent confidence interval is 0.05 and 0.203 . These t-test values indicate there is no significant difference in the radiobiological results of the two mediums of calculation.

\section{Discussion}

Past clinical experience in radiotherapy dose calculation is based on water. However, there have been compelling arguments both in support and against the use of water as a medium of dose reporting and prescription. As per AAPM TG 105 recommendation ${ }^{11}$ both $D_{m}$ and $D_{w}$ should be available for dose calculation and reporting. However, the implications of using $D_{w}$ and $D_{m}$ must be known and understood for specific clinical implications. Based on literature $^{12-13}$ it is known that conversion of $D_{m}$ to $D_{w}$ introduces a systematic error of up to $5.8 \%$ for head and neck and $8 \%$ for prostate cases due to the involvement of bony structures in femoral heads, mandible, and spinal cord. The idea to calculate the dose to water is supported by the fact that there are vast clinical data and a lot of experience obtained with this method. In the current study, the mean difference were $0.62,1.33,3.1$, and $2.3 \%$ for the dose to $0.1 \mathrm{cc}$ of the rectum, small bowel, right, and left femoral heads respectively. Similarly, the mean difference were $0.8,1.22,2.7$, and $2.2 \%$ for the dose to $2 \mathrm{ccs}$ of the rectum, small bowel, right, and left femoral heads respectively. A large systematic error was observed in the femoral head dose due to its bony content. Small systematic differences were observed in OARs like the rectum and small bowel as they contain soft tissues and no large density tissues. 
Table 5. Average of NTCP and TCP Values Obtained from Radiobiological Analysis

\begin{tabular}{|c|c|c|}
\hline & OAR/Target Endpoints & Average NTCP or TCP values Obtained \\
\hline \multirow{6}{*}{ Water (NTCP) } & Rectalx bleeding & 21.44 \\
\hline & Rectal bleeding & 2.3 \\
\hline & Proctitis X & 24.67 \\
\hline & Proctitis & 6.67 \\
\hline & Rectal X Urgency & 32.15 \\
\hline & Rectal Urgency & 13.7 \\
\hline \multirow{6}{*}{ Medium (NTCP) } & Rectalx bleeding & 20.09 \\
\hline & Rectal bleeding & 2.046 \\
\hline & Proctitis X & 23.75 \\
\hline & Proctitis & 6.22 \\
\hline & Rectal X Urgency & 30.99 \\
\hline & Rectal Urgency & 12.92 \\
\hline Water NTCP) & \multirow{2}{*}{ Small Bowel Complication } & 6 \\
\hline Medium (NTCP) & & 5.62 \\
\hline Water (TCP) & \multirow{2}{*}{ PTV_Primary } & 81.24 \\
\hline Medium (TCP) & & 79.74 \\
\hline
\end{tabular}

Rectal bleeding, Proctitis, and Rectal Urgency are NTCP probability for grade 2

Rectalx bleeding, Proctitisx and Rectalx Urgency are combined NTCP probability for grade 1 and 2

In Monaco conversion of $D_{m}$ to $D_{w}$ with clinically accepted plans involves a simple calculation with the same Monitor Units and other plan parameters. The mass stopping power ratio is applied in each voxel. From our data, we found that there was a very small difference between the $D_{w}$ and $D_{m}$ for low-density regions. This is because for the low-density region the stopping power ratio is close to 1 and hence it will have a minimum effect on the results in Equation 1, whereas for a high-density region like femoral heads which consists of bone the systematic error is as high as $7.4 \%$. The maximum difference was less than $1 \%$ and $2 \%$ for rectum and small bowel respectively. This small difference between the results of the calculation in water and medium might be significant when the OAR receives a dose close to its tolerance dose. Our results are in complete conformity with previous studies. Chen et $\mathrm{al}^{14}$ evaluated different sites to understand the consequence of prescription in 2 different media i.e., $D_{m}$ and $D_{w}$. The $D_{m}$ versus $D_{w}$ comparison was based upon dose-volume indices and it was concluded that the difference between $D_{m}$ and $D_{w}$ has a very little clinical impact. However, the plans in which a certain OAR/Target dose is just close to its tolerance/prescription and is situated near bony structures the selection of $D_{m}$ versus $D_{w}$ may have a consequential impact on the quality of the plan. Sarvana et $\mathrm{al}^{15}$ in their retrospective study determined that there is no significant difference between the dosimetric results obtained from $D_{m}$ and $D_{w}$ mode of calculation. There are also studies where there are opposite conclusions Usmani et $\mathrm{al}^{16}$ compared the absorbed $\mathrm{D}_{\mathrm{m}}$ and $\mathrm{D}_{\mathrm{w}}$ for the spine IMRT plan using a commercial Monte Carlo treatment planning system. A systematic difference between dose volumes indices like $\mathrm{D}_{2}, \mathrm{D}_{50}$, and $\mathrm{D}_{98}$ of CTV was observed. They concluded that dose to water is a better quantity for dose prescription in photon beam treatment planning using MC systems. They also determined that it was appropriate to report $\mathrm{D}_{\mathrm{m}}$ for critical structures. Walters et $\mathrm{al}^{17}$ concluded that it is better to specify $D_{w}$ rather than $D_{m}$ in Monte Carlo treatment plans, since $D_{w}$ provides a better estimate of dose to sensitive tissue in bone, as it is the only location where the difference between the two quantities is significant.

In Table 3 we have presented a comparison of the 2 modes of calculation for the target. The average difference between target dose is $0.3 \%$ (percent points). The maximum difference observed in the target was $1.6 \%$ for $\mathrm{D}_{98}$. The difference is very small as it neither involves any air soft tissue interface nor high-density bone content. Based on this we can understand that certain clinical situations for the target like bone metastasis or hotspot in the bone can make optimization more complex. In Figure 1 the DVH value for the modes of calculation i.e., $D_{w}$ and $D_{m}$ have been provided for one case.

Table 5 presents the radiobiological comparison of the dose received by the OARs and target. This evaluation involves different grades of toxicity. For example, in the case of rectal bleeding, the NTCP has been calculated for grades 1 and 2 combined and then grade 2 separately as well. A similar calculation has been done for proctitis and rectal bleeding. A similar calculation has been done to determine the NTCP for small bowel complications using the LKB model for OAR. In Table 5 average TCP for target has been provided using the target Poisson model for PTV. The mean percentage difference between NTCP in $D_{w}$ versus $D_{m}$ mode was $9.65 \%$ with a maximum percentage difference of $25 \%$. The mean percentage difference in NTCP of the rectum for the two modes of calculation were $0.97,1.22$, and $6.85 \%$ for grade 1 and 2 combined toxicities like rectal bleeding, proctitis, and rectal urgency. For grade 2 toxicity the mean percentage difference between NTCP for $D_{w}$ and $D_{m}$ modes were $0.26,0.46$, and $0.81 \%$ for toxicities like rectal bleeding, proctitis, and rectal urgency. 


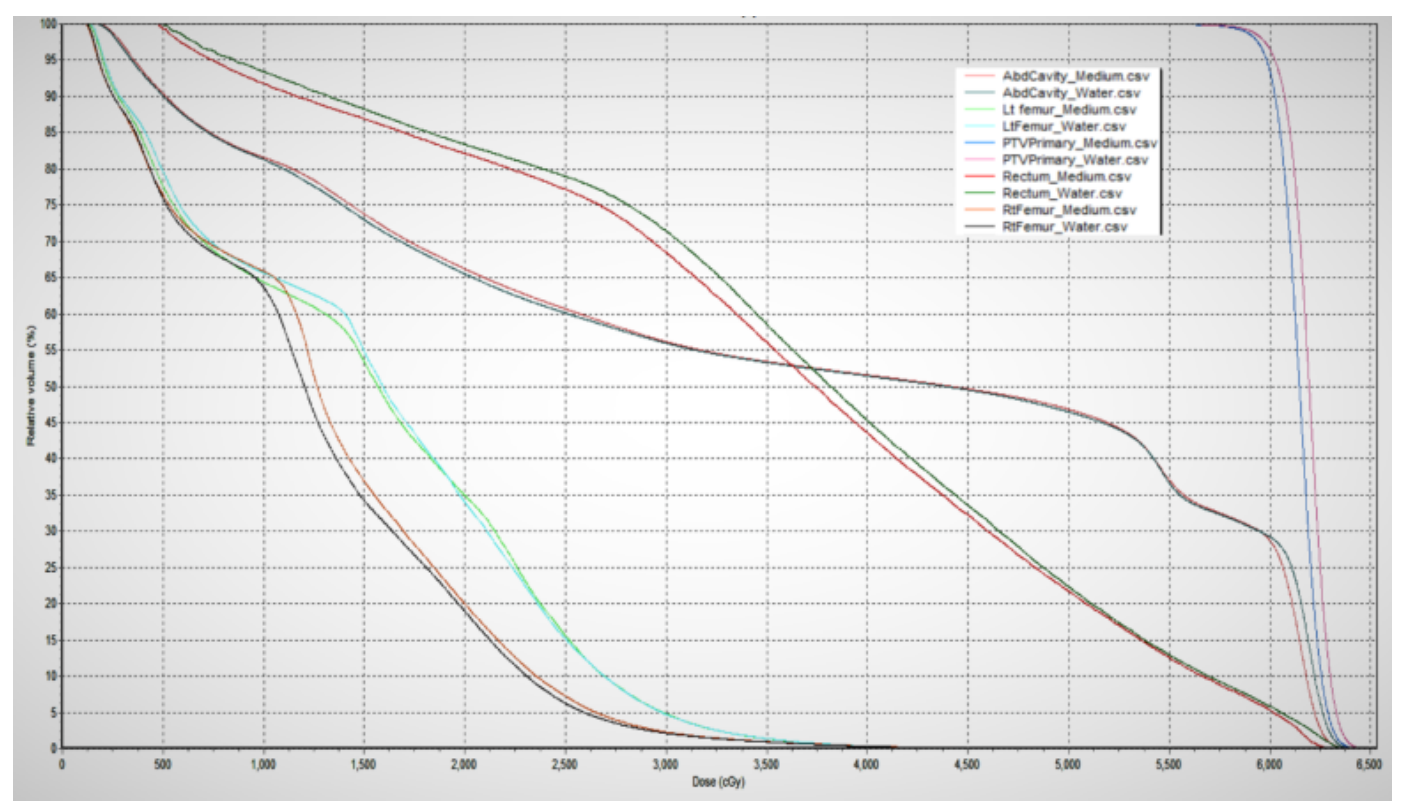

\section{Figure 1. DVH for a patient in both modes of calculation i.e., dose to water and dose to medium}

The corresponding maximum NTCP difference in the rectum were $7.1,11.3$ and $14.2 \%$ and $1.3,2.2$, and $7.4 \%$ for grades 1 and 2 combined and grade 2 toxicity respectively. The mean percentage difference of TCPs calculated with two modes was $1.90 \%$ and the maximum difference is $9.25 \%$.

Both models have different advantages as suggested by their proponents. ${ }^{18}$ Monte Carlo has a unique advantage of calculation in the actual medium and the sheer desire to comply with tradition may be a reason to report $\mathrm{D}_{\mathrm{w}}$. The most important feature of Monte Carlo is its accuracy. But computing stopping power ratios of each voxel and applying it adds nothing but uncertainty and systematic error to the dose calculation in addition to making it more time-consuming. The impact of calculation in dose to medium is very small or insignificant and hence the difference between $D_{w}$ and $D_{m}$ will not change clinical outcomes to a noticeable degree as long as the dose received by OAR is very close to its tolerance. For target, it might also some influence if the dose calculated with one method is close to the prescribed dose.

The NTCP and TCP values obtained by the study are based upon the parameter in radiobiological models which have been derived based on the experience in $\mathrm{D}_{\mathrm{w}}$ studies and its direct application to the medium can be questioned. This is an inherent limitation of our study.
The proponents of $\mathrm{D}_{\mathrm{m}}$ challenge the above arguments by using the fact that the relationship is known between the doses to water and medium and the required number of photon histories to be simulated. ${ }^{19}$ From a prescription of $D_{m}$ in a patient, the corresponding number of photon histories can be converted to monitor units $(\mathrm{mu})$ through the use of the calibrated value of $D_{w}$ per mu. Hence the calculation of MU can proceed in the same way for $D_{w}$ or $D_{m}$ in Monte Carlo simulation. The fact is for cells embedded in a heterogeneous medium like in lungs neither $D_{w}$ nor $D_{m}$ gives the accurate dose because the CT image resolution is unable to determine structures smaller than a voxel and $D_{w}$ or $D_{m}$ just gives an averaged dose over a voxel. So, both $D_{w}$ and $D_{m}$ can be used to calculate the dose delivered and subsequent radiobiological effect.

\section{Conclusions}

It was found that the difference between dose to water and medium is less in critical organs like the rectum and small bowel. The same cannot be said about the femoral head with its bony content. The radiobiological difference between NTCP of the OARs like the rectum, small bowel and femur are not significant. That result might be important when the OARs are receiving a dose very close to their tolerance dose.

\section{References}

1. Schoknecht G. Description of radiation fields by separation of primary and scatter radiation. I. The tissue-air ratio in 60 Co fields. Strahlentherapie. 1967:132:516-528.

2. Batho HF. Lung corrections in Cobalt 60 Beam Therapy. J Can Assoc Radiol. 1964;15:79-83.

3. Oelkfe U, Scholz C. Dose Calculation Algorithms. In: Schlegel W., Bortfeld T., Grosu AL. (eds) New Technologies in Radiation Oncology. Medical Radiology (Radiation Oncology). Springer, Berlin, Heidelberg. 2006. https://doi.org/10.1007/3-540-29999-8_15

4. Siebers JV, Keall PJ, Nahum AE, Mohan R. Converting absorbed dose to medium to absorbed dose to water for Monte Carlo based photon beam dose calculations. Phys Med Biol. 2000;45(4):983-95. https://doi.org/10.1088/0031-9155/45/4/313 
5. Almond PR, Biggs PJ, Coursey BM, et al. AAPM's TG-51 protocol for clinical reference dosimetry of high-energy photon and electron beams. Med Phys. 1999;26(9):1847-1870. https://doi.org/10.1118/1.598691

6. Uzan J, Nahum AE. Radiobiologically guided optimisation of the prescription dose and fractionation scheme in radiotherapy using BioSuite. Br J Radiol. 2012;85(1017):1279-1286. https://doi.org/10.1259/bjr/20476567

7. Lyman JT. Complication probability as assessed from dose-volume histograms. Radiat Res Suppl. 1985;8:S13-S19. https://doi.org/10.2307/3583506

8. Gulliford SL, Partridge M, Sydes MR, et al. Parameters for the Lyman Kutcher Burman (LKB) model of Normal Tissue Complication Probability (NTCP) for specific rectal complications observed in clinical practise. Radiother Oncol. 2012;102(3):347-351. https://doi.org/10.1016/j.radonc.2011.10.022

9. Burman C, Kutcher GJ, Emami B, Goiten M. Fitting of normal tissue tolerance data to an analytic function. Int J Radiat Oncol Biol Phys. 1991;21:123-35. https://doi.org/10.1016/0360-3016(91)90172-Z

10. Lutkenhaus LJ, Vestergaard A, Bel A, et al. A biological modeling based comparison of two strategies for adaptive radiotherapy of urinary bladder cancer. Acta Oncologica. 2016;55(8):1009-1015. :8, 1009-1015. https://doi.org/10.3109/0284186X.2016.1151548

11. Chetty IJ, Curran B, Cygler JEet al. Report of the AAPM Task Group No.105: Issues associated with clinical implementation of Monte Carlo-based photon and electron external beam treatment planning. Med Phys. 2007;34:4818-53. https://doi.org/10.1118/1.2795842

12. Dogan N, Siebers JV, Keall PJ. Clinical Comparison of Head and Neck and Prostate IMRT Plans Using Absorbed Dose to Medium and Absorbed Dose to Water. Phys Med Biol. 2006;51(19):4967-4980. https://doi.org/10.1088/0031-9155/51/19/015

13. Siebers JV, Keall PJ, Nahum AE, Mohan R. Converting Absorbed Dose to Medium to Absorbed Dose to Water for Monte Carlo Based Photon Beam Dose Calculation. Phys Med Biol. 2000;45(4):983-995. https://doi.org/10.1088/0031-9155/45/4/313

14. Chen L, Huang B, Huang X, et al. Clinical evaluation for the difference of absorbed doses calculated to medium and calculated to water by Monte Carlo method. Radiat Oncol. 2018;13:137. https://doi.org/10.1186/s13014-018-1081-3

15. Gopal SK, Dash PC. Dose-to-medium vs. dose-to-water: Dosimetric evaluation of head and neck VMAT cases using Monaco treatment planning system. Int J Cancer Ther Oncol. 2016;4(4):4416. https://doi.org/10.14319/ijcto.44.16

16. Usmani M, Masai N, Oh R, et al. Comparison of Absorbed Dose to Medium and Absorbed Dose to Water for Spine IMRT Plans Using a Commercial Monte Carlo Treatment Planning System. International Journal of Medical Physics, Clinical Engineering and Radiation Oncology. 2014;3(1):60-66. http://dx.doi.org/10.4236/ijmpcero.2014.31010

17. Walters BRB, Kramer R, Kawrakow I. Dose to medium versus dose to water as an estimator of dose to sensitive skeletal tissue. Phys Med Biol. 2010;55:4535. https://doi.org/10.1088/0031-9155/55/16/S08

18. Fippel M, Nüsslin F. Comments on 'Converting Absorbed Dose to Medium to Absorbed Dose to Water for Monte Carlo Based Photon Beam Dose Calculations. Phys Med Biol. 2007;45(8):L17-L19. https://doi.org/10.1088/0031-9155/45/8/101

19. Ma CM, Mok E, Kapur A, et al. Clinical Implementation of a Monte Carlo Treatment Planning System. Med Phys. 1999;26(10):2133-2143. https://doi.org/10.1118/1.598729

20. Keall P. Dm Rather than Dw Should Be Used in Monte Carlo Treatment Planning. Against the Proposition. Med Phys. 2002;29(5):923-924. https://doi.org/10.1118/1.1473137 